\title{
CAMELLIA THUONGIANA - A NEW YELLOW CAMELLIA SPECIES FROM VIETNAM
}

\author{
Luong Van Dunga*, Le Annab, Nguyen Thi Hanha, Nguyen Thi Lieu ${ }^{\mathrm{a}}$ \\ ${ }^{a}$ The Faculty of Biology, Dalat University, Lamdong, Vietnam \\ ${ }^{b}$ Kim Hoa Tra Company, Lamdong, Vietnam \\ Article history \\ Received: August 03 ${ }^{\text {rd }}, 2016$ | Received in revised form: August $26^{\text {th }}, 2016$ \\ Accepted: August 29 ${ }^{\text {th }}, 2016$
}

\begin{abstract}
Camellia thuongiana Luong, Anna Le \& Lau (Theaceae), a new yellow Camellia species from Doanket commune, Dahuoai district, Lamdong province, is described and illustrated. Based on morphological data we affirm that this new species belong to section Chrysantha.
\end{abstract}

Keywords: Camellia; Lamdong; New species; Theaceae; Vietnam.

\section{INTRODUCTION}

The yellow camellias are the species of Camellia L. genus (Theaceae). There are about 40 yellow Camellia species in Vietnam. In Lamdong, the yellow species are: Camellia capitata Orel, Curry \& Luu; Camellia dalatensis V. D. Luong, Tran \& Hakoda; Camellia dilinhensis Ninh \& V. D. Luong; Camellia dormoyana (Pierre ex Laness) Sealy; Camellia inusitata Orel, Curry \& Luu; Camellia luteopallida Luong, T. Q. T. Nguyen \& Luu; Camellia ninhii Luong \& Le and Camellia vidalii Rosmann (Le \& Luong, 2016). During a field trip in Doanket commune, Dahuoai district, Lamdong province in January 2016, we obtained some yellow Camellia samples. After analyzing and comparing morphological characteristics with closely species, we assert that this is a new species, named as Camellia thuongiana Luong, Anna Le \& Lau.

\section{MATERIAL AND METHODS}

Wild individuals of this species were found in Doanket commune, Dahuoai district, Lamdong province, Southern Vietnam in January 2016. Specimens were deposited in the Herbarium of Dalat University (DLU) with $\mathrm{N}^{0}$ : DL.160107, 
DL.160108, DL.160109. Using comparative morphology method, we have classified the species in the genus Camellia in general and yellow Camellia species in particular.

\section{RESULTS}

\subsection{Characteristics of Camellia thuongiana Luong, Anna Le \& Lau, sp. nov.}

Figures 1 and 2 show the characteristics of Camellia thuongiana Luong, Anna Le \& Lau, sp. nov. as follows: Small tree, 3-5 m. high, evergreen; young branches dark dull red, hairy and soon becoming glabrous. Leaves stalked; blades elliptic to oblong elliptic, 9-17 $\mathrm{cm}$. long and 4-6.5 $\mathrm{cm}$. wide, apex acuminate to long caudate, base broadly cuneate or nearly rounded, margin shallowly serrulate; mature leaves coriaceous, shiny on both sides, venation prominent below, lateral vein 12-13 pairs; petioles 8-11 mm. long, glabrous. Flowers $4.5-5 \mathrm{~cm}$. in diameter, solitary or 2-3(4) at the ends of branches or axils of leaves. Pedicel 8-10 mm. long, glabrous; bracteoles 3-4, dentate to elliptic, $2-4.5 \mathrm{~mm}$. long, $2-4 \mathrm{~mm}$. wide, glabrous on both sides, margin ciliolate, bracteoles caducous. Sepals 5, semiorbicular to suborbicular, 6-10 mm. long, 7-11 mm. wide, glabrous on both sides, sepals persistent. Petals 11-13, bright yellow, suborbicular to elliptic or obovate; 4-5 outer petals, suborbicular to elliptic, $1.4-1.9 \mathrm{~cm}$. long, 1.3-1.5 cm. wide, glabrous on both sides; 7-8 inner petals, elliptic or obovate, 1.9$2.2 \mathrm{~cm}$. long, 1.3-1.4 cm. wide, united with outermost filaments at the base, glabrous on both sides. Androecium 290-340 stamens, in 6-7 circles; filaments 1.3-1.4 cm. long, glabrous, outer filament whorl basally connate for 4-5 $\mathrm{mm}$. Gynoecium 3 loculi, ovary ovoid, pubescent, 4-4.5 mm. high, 4-5 mm. wide; styles 3 , free to $1 / 2$ from the base, $8-9$ $\mathrm{mm}$. long, glabrous. Capsule not seen.

Blooming season: Winter to spring.

This species was found in evergreen broadleaved forest or mixed wood-bamboo forest of Dahuoai district (Lamdong province), at altitudes of 500-800m.

Typus: Doanket commune, Dahuoai district, Lamdong province, Southern Vietnam, Lau Quoc Thuong, Luong Van Dung, DL.160107 (Holotype, DLU; Isotype, DLU); DL.160108 (Paratype, DLU). 
Etymology: The specific epithet of this species honors Mr. Lau Quoc Thuong, who first discovered samples of this species.

\subsection{The key of yellow Camellia species in Lamdong}

1a. Leaves more than $35 \mathrm{~cm}$. long

2a. Ovary 4-5 loculi

3a. Leaves pubescent

1. Camellia dalatensis

3b. Leaves glabrous

2. Camellia vidalii

2b. Ovary 3 loculi

3. Camellia capitata

1b. Leaves less than $35 \mathrm{~cm}$. long

4a. Ovary 5 loculi

4. Camellia dormoyana

4b. Ovary 3 loculi

5a. Ovary glabrous

5. Camellia luteopallida

5b. Ovary pubescent

6a. Young branches flat.

6. Camellia inusitata

6b. Young branches rounded

7a. Leaves pubescent

7. Camellia ninhii

7b. Leaves glabrous

8a. Ovary cylinder; styles pubescent

8. Camellia dilinhensis

8b. Ovary ovoid; styles glabrous

9. Camellia thuongiana

After examining the specimens and comparing with the Camellia species in Vietnam and Lamdong, we conclude that the specimens collected from Doanket commune are similar to Camellia dilinhensis (Tran \& Luong, 2013) and Camellia ninhii (Luong \& Le, 2016) in certain characters. The main similar and differences between the new species with Camellia dilinhensis and Camellia ninhii are summarized in Table 1.

Camellia thuongiana resemble especially Camellia ninhii of sect. Chrysantha Chang (Chang \& Bartholomew, 1984) and Camellia dilinhensis of sect. Obvoidae Tran et Luong (Tran \& Luong, 2013) by having the following characteristics: oblong elliptic or elliptic leaves; flower yellow, solitary or a pair at the ends of branches, sepals 5 , persistent, petals 8-12, filaments glabrous; ovary 3 loculi, pubescent; style 3, free. 
Table1. Morphological comparison between C.dilinhensis, $C$.ninhii with C.thuongiana

\begin{tabular}{|c|c|c|c|}
\hline Characters & C.dilinhensis & C.ninhii & C.thuongiana \\
\hline $\begin{array}{l}\text { Leaf blade } \\
\text { shape }\end{array}$ & $\begin{array}{l}\text { Oblong elliptic or elliptic, } \\
\text { glabrous }\end{array}$ & $\begin{array}{l}\text { Elliptic to oblong elliptic, } \\
\text { pubescent below }\end{array}$ & $\begin{array}{l}\text { Elliptic to oblong elliptic, } \\
\text { glabrous }\end{array}$ \\
\hline Leaf size & $\begin{array}{l}\text { 16-24.5 cm. long, } 5.5-9.5 \\
\mathrm{~cm} . \text { wide }\end{array}$ & $\begin{array}{l}\text { 14-20 cm. long, } 5-7 \mathrm{~cm} . \\
\text { wide }\end{array}$ & $\begin{array}{l}\text { 9-17 cm. long, } 4-6.5 \mathrm{~cm} \text {. } \\
\text { wide }\end{array}$ \\
\hline Leaf apex & Acuminate & Acuminate to nearly caudate & Acuminate to long caudate \\
\hline Leaf base & Cuneate & $\begin{array}{l}\text { Broadly cuneate or nearly } \\
\text { rounded }\end{array}$ & $\begin{array}{l}\text { Broadly cuneate or nearly } \\
\text { rounded }\end{array}$ \\
\hline Petiole & $1-1.9 \mathrm{~cm}$. long, glabrous & $\begin{array}{l}\text { 4-7 mm. long, } \\
\text { hirsute }\end{array}$ & $\begin{array}{l}\text { 8-11 mm. long, } \\
\text { glabrous }\end{array}$ \\
\hline Flowers & $\begin{array}{l}1-3 \text { in each group in } \\
\text { axillary }\end{array}$ & $\begin{array}{l}\text { Solitary or a pair at the ends } \\
\text { of branches }\end{array}$ & $\begin{array}{l}\text { Solitary or 2-3(4) at the } \\
\text { ends of branches or axils } \\
\text { of leaves }\end{array}$ \\
\hline $\begin{array}{l}\text { Flower } \\
\text { diameter }\end{array}$ & $3.5-3.7 \mathrm{~cm}$. & $3-3.5 \mathrm{~cm}$. & $4.5-5 \mathrm{~cm}$ \\
\hline Pedicel & 5-7 mm. long, glabrous & 2-3 mm. long, glabrous & 8-10 mm. long, glabrous \\
\hline Bracteoles & $2-3$ & & $3-4$ \\
\hline $\begin{array}{l}\text { Sepal } \\
\text { number }\end{array}$ & 5 & $6-7$ & 5 \\
\hline Sepal size & $\begin{array}{l}4-8 \mathrm{~mm} \text {. long, } 8-11 \mathrm{~mm} \text {. } \\
\text { wide }\end{array}$ & 2-7 mm. long, 3-9 mm. wide & $\begin{array}{l}\text { 6-10 mm. long, 7-11 mm. } \\
\text { wide }\end{array}$ \\
\hline $\begin{array}{l}\text { Sepal } \\
\text { hairiness }\end{array}$ & Glabrous & Finely puberulous inside & Glabrous \\
\hline $\begin{array}{l}\text { Petal } \\
\text { number }\end{array}$ & $8-9$ & $9-11$ & $11-13$ \\
\hline Petal shape & Nearly rounded to elliptic & Nearly rounded to elliptic & $\begin{array}{l}\text { Suborbicular to elliptic or } \\
\text { obovate }\end{array}$ \\
\hline Petal size & $\begin{array}{l}1.5-1.7 \mathrm{~cm} \text {. long, } 1.2-1.4 \\
\mathrm{~cm} \text {. wide }\end{array}$ & $\begin{array}{l}0.5-1.9 \mathrm{~cm} . \text { long, } 0.9-1.3 \mathrm{~cm} \text {. } \\
\text { wide }\end{array}$ & $\begin{array}{l}1.4-2.2 \mathrm{~cm} \text {. long, } 1.3-1.5 \\
\mathrm{~cm} . \text { wide }\end{array}$ \\
\hline $\begin{array}{l}\text { Pepal } \\
\text { hairiness }\end{array}$ & Glabrous & Finely puberulous inside & Glabrous \\
\hline Stamens & 350 stamens, $4-5$ circles & 200-300 stamens, 5-6 circles & $\begin{array}{l}290-340 \text { stamens, } 6-7 \\
\text { circles }\end{array}$ \\
\hline Filaments & $\begin{array}{l}5-9 \text { mm. long, } \\
\text { glabrous }\end{array}$ & 4-10 mm. long, glabrous & 1.3-1.4 cm. long, glabrous \\
\hline Ovary & $\begin{array}{l}\text { Cylinder, } 3 \text { loculi, } \\
\text { pubescent }\end{array}$ & Ovoid, 3 loculi, pubescent & Ovoid, 3 loculi, pubescent \\
\hline Style & $\begin{array}{l}\text { Styles 3, free to the base; } 5 \\
\mathrm{~mm} \text {. long, pubescent }\end{array}$ & $\begin{array}{l}\text { Styles } 3 \text {, free to the base, } 8-9 \\
\text { mm. long, glabrous }\end{array}$ & $\begin{array}{l}\text { Styles } 3 \text {, free to } 1 / 2 \text { from the } \\
\text { base, } 8-9 \mathrm{~mm} \text {. long, } \\
\text { glabrous }\end{array}$ \\
\hline
\end{tabular}


However, Camellia thuongiana is distinct in having 3-4 bracteoles $(C$. dilinhensis have 2-3 bracteoles, C.ninhii have 6-7 bracteoles and sepals); sepals and petals glabrous ( $C$. ninhii finely puberulous inside); ovary ovoid ( $C$. dilinhensis cylinder); style 3 , free to $1 / 2$ from the base, glabrous $(C$. dilinhensis styles 3 , free to the base, pubescent). Camellia thuongiana also has some similar characteristics with other species of section Chrysantha, and possesses typical characteristics of Camellia sect. Chrysantha Chang (Chang \& Bartholomew, 1984), such as flower have pedicel, sepals 5-7, petals more than 5, yelow, androecium glabrous, gynoecium 3 with styles 3 , free, glabrous (Camellia crassiphylla, Camellia euphlebia, Camellia hulungensis (Tran, 2002, 2003). For all reasons, it would be properly to classify this species into Camellia section Chrysantha Chang.

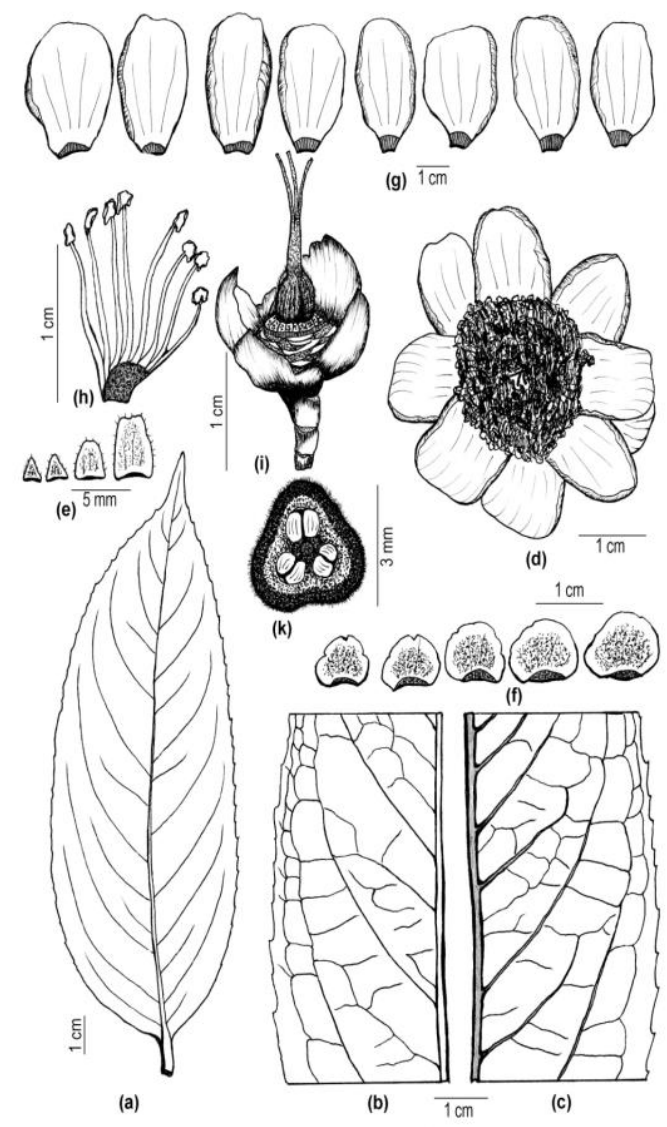

Figure 1. Camellia thuongiana Luong, Anna Le \& Lau

Note: (a) Leaf; (b) Part of upper surface of leaf; (c) Part of lower surface of leaf; (d) Flower; (e) Bracteoles (inner surface); (f) Sepals (inner surface); (g) Petals (inner surface);

(h) Filaments; (i) Calyx and gynoecium; (k) Ovary (cross-section).

(Drawn by Luong Van Dung) 


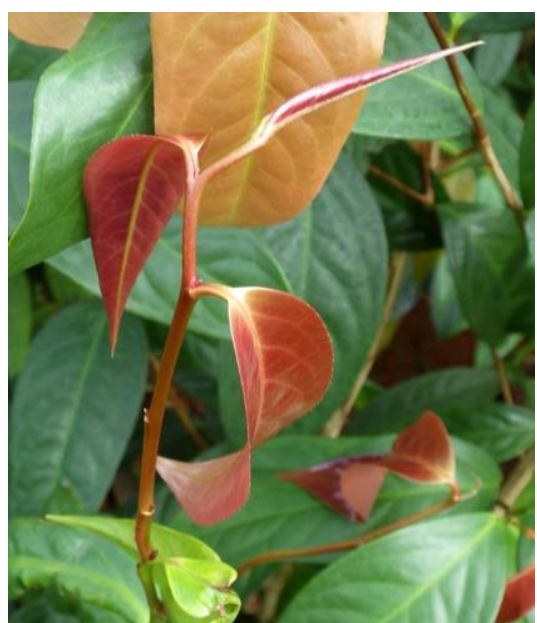

(a)

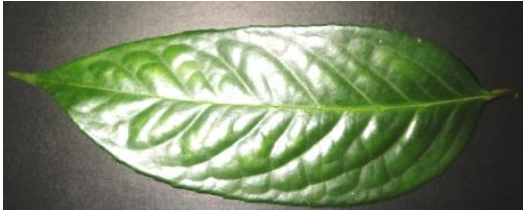

(b)

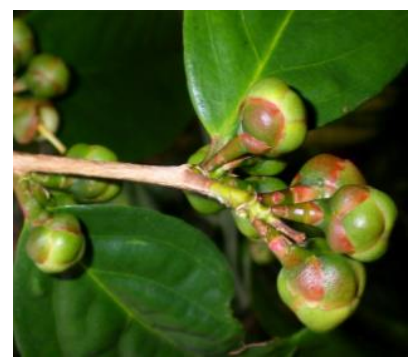

(c)

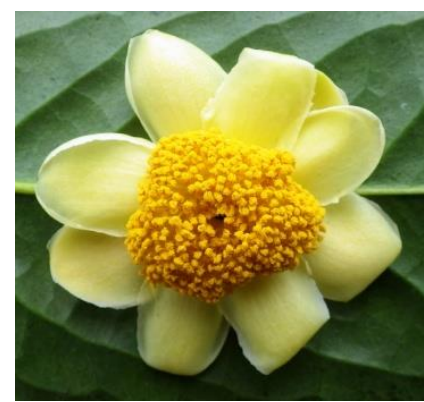

(d)

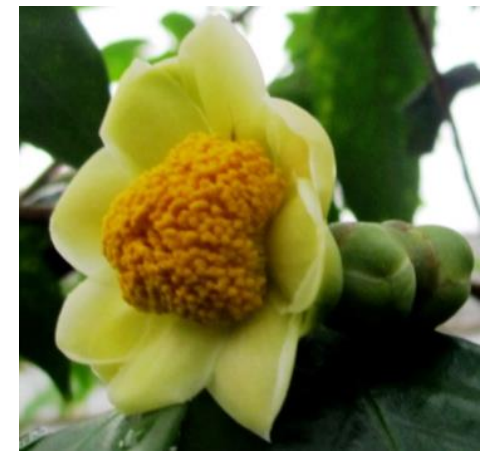

(e)

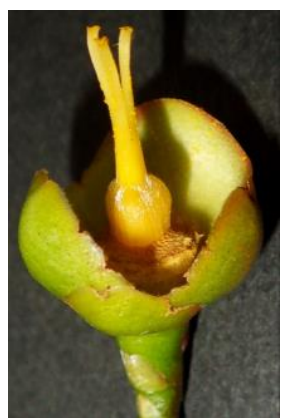

(f)

Figure 2. Camellia thuongiana Luong, Anna Le \& Lau

Note: (a) Young twig; (b) Leaf; (c) Flower buds; (d,e) Flower; (f) Calyx and gynoecium

\section{REFERENCES}

Chang, H. T. \& Bartholomew, B. (1984). Camellias. Portland: Timber Press.

Le, N. H. N. \& Luong, V. D. (2016). General information about the Yellow Camellia Species in Vietnam. Proceedings of Dali International Camellia Congress, Dali, Yunnan, China.

Luong, V. D. \& Le, N. H. N. (2016). Camellia Ninhii - A new Yellow Camellia Species from Vietnam. Proceedings of Dali International Camellia Congress, Dali, Yunnan, China.

Tran, N. (2002). Biodiversity of Camellia genus of Viet Nam. Proceedings of The first National Symposium on Yellow Camellia of Viet Nam, Tam Dao, Viet Nam.

Tran, N. (2003). Results of the study on yellow Camellias of Vietnam. International Camellia Journal, 35, 73-75.

Tran, N. \& Luong, V. D. (2013). Camellia dilinhensis - A new Yellow Species from Vietnam. International Camellia Journal, 45, 87-89. 


\title{
CAMELLIA THUONGIANA - MỘT LOÀI TRÀ MI HOA VÀNG MỚI CỦA VIẸTT NAM
}

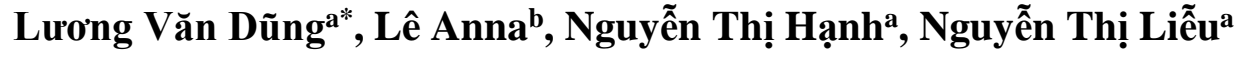 \\ ${ }^{a}$ Khoa Sinh học, Truoòng Đại học Đà Lạt, Lâm Đồng, Việt Nam \\ ${ }^{b}$ Công ty Kim Hoa Trà, Lâm Đồng, Việt Nam \\ *Tác giả liên hệ: Email: dunglv@dlu.edu.vn
}

\section{Lịch sử bài báo}

Nhận ngày 03 tháng 08 năm 2016|Chỉnh sửa ngày 26 tháng 08 năm 2016

Chấp nhận đăng ngày 29 tháng 08 năm 2016

\section{Tóm tắt}

Trà mi Thưởng (Camellia thuongiana Luong, Ann Le \& Lau) thuộc họ Chè (Theaceae) đã được mô tả và vẽ hình, đây là một loài trà mi hoa vàng mói, phân bố ở xã Đoàn Kết, huyện Đạ Huoai, tỉnh Lâm Đồng. Trên cơ sở dũ liệu hình thái xác nhận loài mói thuộc sect. Chrysantha.

Từ khóa: Camellia; Lâm Đồng; Loài mới; Theaceae; Việt Nam. 